\title{
What will be the role of molnupiravir in the treatment of COVID-19 infection?
}

\author{
Antonio Vitiello $^{1}$ (1) $\cdot$ Valentina Troiano $^{1} \cdot$ Raffaele La Porta $^{1}$
}

Accepted: 25 October 2021 / Published online: 5 November 2021

(c) The Author(s), under exclusive licence to Springer Nature Switzerland AG 2021

The global COVID-19 pandemic is currently underway. To date, there are approximately 4.55 million deaths and 219 million people infected [1]. The massive vaccination campaign is continuing, representing an important weapon to stop the COVID-19 pandemic. Since the pandemic began, in addition to COVID-19 vaccine development, several pharmacological treatments have been studied [2-4]. One antiviral treatment that has shown good results is remdesivir [5-7]. However, some clinical trials have failed to fully confirm its beneficial effects against Sars-CoV-2, and, in addition, the drug is expensive and must be administered intravenously in a hospital setting. Recently, other antiviral alternatives are demonstrating effective results in preregistration studies. Among them, molnupiravir, an oral antiviral agent, has emerged as a promising new drug that molecularly targets the RNA polymerase of Sars-CoV-2. The RNA polymerase are key enzymes in the viral replication cycle. Molnupiravir is the isopropyl prodrug of the ribonucleoside analogue $\beta$-D-N4-hydroxycytidine (NHC) [8]. In vitro evidence shows that molnupiravir was found to be a potent inhibitor of SARS-CoV-2 replication, with an $\mathrm{EC}_{50}$ in the submicromolar range [9]; this viral injection effect was also observed in animal models [10]. In addition, molnupiravir in phase I/II/III clinical trials has demonstrated good efficacy and safety. Data indicate that molnupiravir reduced the risk of hospitalization or death by approximately $50 \%$ in non-hospitalized adults who had mild to moderate COVID-19 and were at risk for a serious disease outcome [11]. In addition, molnupiravir has shown to reduce the risk of hospitalization or death in all subgroups, with efficacy unaffected by the timing of symptom onset, underlying risk factors, or type of Sars-CoV-2 variant (gamma, delta, and $\mathrm{mu}$ ). Another positive aspect is that the data demonstrate

Antonio Vitiello

antonio.vitiello2@uslumbria1.it

1 Pharmaceutical Department Usl Umbria 1, Via XIV

Settembre-75, Perugia, Italy a generally good safety profile. The clinical study showed that the incidence of any adverse event was comparable in the molnupiravir and placebo groups (35\% and $40 \%$, respectively), and that the incidence of drug-related adverse events was also comparable ( $12 \%$ and $11 \%$, respectively). In addition, fewer subjects discontinued study therapy because of an adverse event in the molnupiravir group compared with the placebo group (1.3\% vs $3.4 \%)$ [11].

Several additional aspects have to be defined for molnupiravir, such as the optimal dose to be administered, the timing of administration and any interactions with other drugs used to manage the COVID-19 infection. The safety profile of molnupiravir also has to be better defined by largescale epidemiological data from the post-marketing phase. Molnupiravir is the first oral, direct-acting antiviral shown to be highly effective at reducing nasopharyngeal SARS-CoV-2 infectious virus and viral RNA and has a favorable safety and tolerability profile. Oral administration of molnupiravir is a big advantage over antiviral injections that work against COVID-19, such as remdesivir. With preliminary analysis showing promising results against COVID-19 infection, future clinical trials are awaited with interest.

\section{Declarations}

Funding None.

Conflicts of interest None of the authors have conflicts of interest to disclose.

Ethical approval Not applicable.

Consent to participate Not applicable.

Consent to publish The authors consent to the publication of the manuscript.

Availability of data and materials Full availability of data and materials. 
Authors contributions AV: conceptualization, writing-original draft, methodology, writing — original draft. VT: validation. RLP: validation.

\section{References}

1. World Health Organization (WHO). World Health Organization Coronavirus disease (COVID-19) weekly epidemiological update. https://www.who.int/emergencies/diseases/novel-coronavirus2019/situation-reports. Accessed 20 Oct 2021.

2. Vitiello A, Ferrara F. Pharmacological agents to therapeutic treatment of cardiac injury caused by COVID-19. Life Sci. 2020;262: 118510.

3. Vitiello A, La Porta R, Ferrara F. Sacubitril, valsartan and SARSCoV-2. BMJ Evid Based Med. 2021;26:205.

4. Francesco F, Antonio V. Efficacy of synthetic glucocorticoids in COVID-19 endothelites. NaunynSchmiedebergs Arch Pharmacol. 2021;394(5):1003-7.

5. Ferner RE, Aronson JK. Remdesivir in COVID-19. BMJ. 2020;369:m1610.

6. Vitiello A, Ferrara F. Remdesivir versus ritonavir/lopinavir in COVID-19 patients. Ir J Med Sci. 2021;190(3):1249-50.
7. Antonio V, Francesco F, La Porta R. Remdesivir and COVID-19 infection, therapeutic benefits or unnecessary risks? Ir J Med Sci. 2021;190(4):1637-8.

8. Agostini ML, Pruijssers AJ, Chappell JD, et al. Small-molecule antiviral $\beta$-D- $N^{4}$-hydroxycytidine inhibits a proofreading-intact coronavirus with a high genetic barrier to resistance. J Virol. 2019;93(24):301348-419.

9. Zhao J, Guo S, Yi D, et al. A cell-based assay to discover inhibitors of SARS-CoV-2 RNA dependent RNA polymerase. Antiviral Res. 2021;190: 105078.

10. Sheahan TP, Sims AC, Zhou S, et al. An orally bioavailable broadspectrum antiviral inhibits SARS-CoV-2 in human airway epithelial cell cultures and multiple coronaviruses in mice. Sci Transl Med. 2021;12(541): eabb5883.

11. Merck and Ridgeback's investigational oral antiviral molnupiravir reduced the risk of hospitalization or death by approximately 50 percent compared to placebo for patients with mild or moderate COVID-19 in positive interim analysis of phase 3 study. https://www.merck.com/news/merck-and-ridgebacks-investigat ional-oral-antiviral-molnupiravir-reduced-the-risk-of-hospitaliz ation-or-death-by-approximately-50-percent-compared-to-place bo-for-patients-with-mild-or-moderat/. Accessed 20 Oct 2021. 